\title{
CORRESPONDENCE
}

\section{Population prevalence of COPD}

\section{To the Editors:}

The study by SHIRTCLIFFE et al. [1] discusses the use of various definitions of abnormal spirometric results to estimate the prevalence of chronic obstructive pulmonary disease in $\mathrm{New}$ Zealand and raises a major question that is not addressed in the analyses.

Childhood asthma is common and can lead to a degree of irreversible airflow obstruction in adulthood. In another New Zealand study, based on the Dunedin longitudinal populationbased cohort, SEARS et al. [2] reported that persistent or relapsing childhood asthma was present in $26.9 \%$ of $26-y r-$ old study subjects. This is not dissimilar to the prevalence of doctor-diagnosed asthma (24\%) in subjects undergoing pulmonary function testing in the Wellington study. RASMUSSEN et al. [3] also reported that airway remodelling, based on an abnormal post-bronchodilator forced expiratory volume in one second/forced vital capacity ratio, determined as a ratio $\geqslant 2 \mathrm{SD}$ below the mean for healthy age- and sex-matched individuals, was present in $22 \%$ of males and $10.5 \%$ of females with asthma at 26 yrs of age.

It is likely, therefore, that a proportion of subjects determined by spirometry to have "chronic obstructive pulmonary disease" in the Wellington study [1] have asthma-related impairment of lung function. The prevalence of nonasthmarelated chronic obstructive pulmonary disease could be determined by excluding subjects with a history of doctordiagnosed asthma, and if so I suspect the actual prevalence of chronic obstructive pulmonary disease, especially in the younger age groups, reported by SHIRTCLIFFE et al. [1] as $5.6 \%$ in those aged $<40$ yrs and $12 \%$ among nonsmokers, would then be significantly lower than reported.

\section{M.R. Sears}

Firestone Institute for Respiratory Health, McMaster University, Hamilton, ON, Canada.

\section{STATEMENT OF INTEREST}

None declared.

\section{REFERENCES}

1 Shirtcliffe P, Weatherall M, Marsh S, et al. COPD prevalence in a random population survey: a matter of definition. Eur Respir J 2007; 30: 232-239.

2 Sears MR, Greene JM, Willan AR, et al. A longitudinal, population-based, cohort study of childhood asthma followed to adulthood. N Engl J Med 2003; 349: 1414-1422.

3 Rasmussen F, Taylor DR, Flannery EM, et al. Risk factors for airway remodeling in asthma manifested by a low postbronchodilator FEV1/vital capacity ratio: a longitudinal population study from childhood to adulthood. Am J Respir Crit Care Med 2002; 165: 1480-1488.

DOI: 10.1183/09031936.00120907

\section{From the authors:}

M.R. Sears is correct in his assumption that a proportion of subjects determined by spirometry to have "chronic obstructive pulmonary disease (COPD)" in our Wellington, New Zealand study have asthma-related impairment of lung function [1]. This comment also applies to all other prevalence surveys using purely spirometric criteria, especially those which base their diagnosis of COPD on pre-bronchodilator values only. We are not aware that other criteria, such as selfreporting of a doctor's diagnosis, a diagnosis based on the presence of respiratory symptoms, or a diagnosis based on other definitions of airflow obstruction, have any greater claim to accuracy in determining prevalence rates. In fact, our study showed that only $17(15 \%)$ out of 116 subjects who met the Global Initiative for Chronic Obstructive Lung Disease (GOLD) criteria had a doctor's diagnosis of COPD. The GOLD definition of COPD is the internationally accepted criteria [2] and is the definition we used for estimating COPD prevalence. It is also the criteria chosen in the recently reported Burden of Obstructive Lung Disease (BOLD) initiative [3].

We calculated the proportion of subjects in the study with asthma. A subject was considered to have asthma if: there was a post-bronchodilator increase in forced expiratory volume in one second $(\mathrm{FEV} 1) \geqslant 15 \%$ predicted [4]; subjects had peak flow variability $\geqslant 20 \%$ during 1 week of testing [4]; subjects had doctor-diagnosed asthma in conjunction with current symptoms (wheeze, or nocturnal shortness of breath and wheeze, or nocturnal chest tightness in the preceding 12 months); or there was doctor-diagnosed asthma in conjunction with inhaler use in the preceding 12 months. Subjects could belong to more than one of these categories.

Out of the 749 study participants, 179 (24\%) met the criteria for a diagnosis of asthma. Of these, 135 (75\%) out of 179 had a doctor's diagnosis, 61 (34\%) met the criteria for peak flow variability and $43(24 \%)$ met the criteria of $15 \%$ reversibility in FEV1. Of the participants in the study with GOLD-defined COPD, 65 (56\%) out of 116 also met the definition of asthma. Of these, 45 (69\%) out of 65 had a doctor's diagnosis of asthma, 32 $(49 \%)$ had peak flow variability and 31 (47.6\%) had $15 \%$ reversibility (these were not mutually exclusive).

The relationship between asthma and COPD is complex. Historically, COPD and asthma have become increasingly differentiated over time [5] and in the "classic" forms of presentation may be easy to separate by the means of structural and physiological findings [6]. However, these 
differences may not be so clear-cut in the clinical setting and there are significant overlaps in clinical, physiological and pathological features of the two diseases, including the mechanisms of bronchial hyperresponsiveness and atopy that drive obstructive phenotypes [7]. Although asthma may progress to chronic irreversible airflow obstruction, changes in reversibility over time are not widely reported $[8,9]$. It may be that we require a new taxonomy to better define the various disorders of airway obstruction.

So in the end perhaps it all comes back to the title of our paper [1]: COPD prevalence... a matter of definition.

\section{P. Shirtcliffe, M. Weatherall and R. Beasley}

Medical Research Institute of New Zealand, Wellington, New Zealand.

\section{STATEMENT OF INTEREST}

None declared.

\section{REFERENCES}

1 Shirtcliffe P, Weatherall M, Marsh S, et al. COPD prevalence in a random population survey: a matter of definition. Eur Respir J 2007; 30: 232-239.
2 Rabe KF, Hurd S, Anzueto A, et al. Global strategy for the diagnosis, management, and prevention of chronic obstructive pulmonary disease: GOLD executive summary. Am J Respir Crit Care Med 2007; 176: 532-555.

3 Buist AS, McBurnie MA, Vollmer WM, et al. International variation in the prevalence of COPD (the BOLD Study): a population-based prevalence study. Lancet 2007; 370: 741-750.

4 Welcome to GINA the Global Initiative For Asthma. www.ginasthma.com Date last accessed: January 30, 2007.

5 Guerra S. Overlap of asthma and COPD. Curr Opin Pulm Med 2005; 11: 7-13.

6 Sciurba FC. Physiologic similarities and differences between COPD and asthma. Chest 2004; 126: Suppl. 2, 117S-124S.

7 Kraft M. Asthma and chronic obstructive pulmonary disease exhibit common origins in any country! Am J Respir Crit Care Med 2006; 174: 238-244.

8 Vonk JM, Jongepier H, Panhuysen CI, Schouten JP, Bleecker ER, Postma DS. Risk factors associated with the presence of irreversible airflow limitation and reduced transfer coefficient in patients with asthma after 26 years of follow up. Thorax 2003; 58: 322-327.

9 Ulrik CS, Backer V. Nonreversible airflow obstruction in lifelong nonsmokers with moderate to severe asthma. Eur Respir J 1999; 14: 892-896.

\section{Right ventricular dysfunction and functional limitation in idiopathic pulmonary fibrosis}

\section{To the Editors:}

We read with great interest the paper by DAL CORSO et al. [1] in a recent issue of the European Respiratory Journal. The authors used a 6-min step test to estimate effort tolerance and exerciserelated oxyhaemoglobin desaturation in patients with interstitial lung disease. Although desaturation with exertion is well accepted as a prognostic tool in patients with interstitial lung disease, conflicting reports exist regarding the distance covered during the test [2-5]. Moreover, evidence is lacking regarding the possible association between cardiac function, exercise capacity and exercise desaturation in patients with interstitial lung disease. In a small cohort of 22 patients (mean \pm SD age $65 \pm 9$ yrs) with well-defined idiopathic pulmonary fibrosis (IPF), we used the 6-min walk test (6MWT) to assess prognosis and to investigate the association of exercise capacity with left and right ventricular echocardiographic parameters. All patients underwent a complete echocardiographic study, including two-dimensional, colour flow, spectral Doppler as well as tissue Doppler imaging for the evaluation of right and left ventricular systolic and diastolic function.

Mean time from initial diagnosis of the disease was 9 months. After a median follow-up period of 22 months, five deaths were recorded (four were due to respiratory failure decompensation and one followed sepsis). A cut-off value of $225 \mathrm{~m}$ for 6MWT distance predicted survival after follow-up with $100 \%$ sensitivity and $60 \%$ specificity (area under curve (AUC) 0.77 , $p=0.01$ ). Moreover, a cut-off value of $>5 \%$ for desaturation with exercise predicted mortality with $58 \%$ sensitivity and $100 \%$ specificity (AUC 0.82, $\mathrm{p}=0.006$ ). Amongst all echocardiographic parameters, 6MWT distance correlated significantly with pulmonary artery systolic pressure $(r=-0.53, p=0.01$; fig. 1$)$, early diastolic peak myocardial velocity of the tricuspid annulus $(\mathrm{r}=-0.45, \mathrm{p}=0.03$; fig. 1$)$ and late diastolic peak myocardial velocity of the tricuspid annulus $(r=-0.44, p=0.03)$. No correlation was found between oxygen desaturation during the $6 \mathrm{MWT}$ and any of the echocardiographic parameters.

Although the number of patients evaluated was small, our results indicate that there is a close association between exercise capacity, indices of right ventricular diastolic function and prognosis in patients with IPF. Exercise capacity in patients with IPF has been shown to be limited mainly by gas exchange and ventilatory impairment [6]. The conflicting reports in the literature regarding the relationship between $6 \mathrm{MWT}$ distance and mortality in IPF patients probably reflect differences in the exercise protocols and, particularly, the time and reason of the 6MWT termination [2-5]. Nevertheless, the 